\title{
TRANSMUTED ESSCHER TRANSFORMED LAPLACE DISTRIBUTION AND ITS APPLICATION TO MICROARRAY ANALYSIS
}

\author{
PUNATHUMPARAMBATH BINDU ${ }^{\star}$ \\ Department of Statistics, Govt. Arts and Science College, Calicut, Kerala, India \\ Email:ppbindukannan@gmail.com
}

\begin{abstract}
SUMMARY
Esscher transformed Laplace distribution is a new class of asymmetric heavy tailed distribution. In this article, we generalize the Esscher transformed Laplace distribution using the quadratic rank transmutation map to develop transmuted Esscher transformed Laplace distribution. We derived the probability density function of transmuted Esscher transformed Laplace distribution and its various properties were studied. The maximum likelihood estimation procedure is employed to estimate the parameters of the proposed distribution and an algorithm in $\mathrm{R}$ package is developed to carry out the estimation. Simulation studies for various choices of parameter values were performed to validate the algorithm. Finally, we fitted the transmuted Esscher transformed Laplace, Esscher transformed Laplace and Gaussian distributions to microarray gene expression dataset and compared them.
\end{abstract}

Keywords and phrases: Laplace distribution, Esscher transformed Laplace distribution, transmuted Esscher transformed Laplace distribution, microarray gene expression

AMS Classification: 60E05 ; 62F10 ; 62P10.

\section{Introduction}

Recently, there is a lot of research interest in developing new generalized families of distributions which have applications in modeling data in many applied areas such as finance, economics, engineering, lifetime analysis and in biomedical research. Shaw and Buckley (2007), introduced new family of distributions transforming cumulative distribution functions (CDF) through the quadratic rank transmutation map and applied to uniform, exponential and normal distributions. Transmuted distributions found applications in many areas for analyzing frequently occurring large scale applied science experimental data.

Recently, many authors used the transmuted-generalized (T-G) family to propose new generalizations of several distributions, for example, transmuted generalized extreme value (Aryal and Tsokos, 2009), transmuted Weibull (Aryal and Tsokos, 2011), transmuted Lindley (Merovci, 2013), transmuted Pareto (Merovci and Pukab, 2014), transmuted Laplace (Hady and Shalabi, 2016) and transmuted Birnbaum-Saunders (Bourguignon et al., 2017).

\footnotetext{
${ }^{\star}$ Corresponding author

(c) Institute of Statistical Research and Training (ISRT), University of Dhaka, Dhaka 1000, Bangladesh.
} 
Esscher transformed Laplace (ETL) distribution is one of the asymmetric generalizations of Laplace distribution. Recently Arezoomand et al. (2018) introduced new asymmetric generalization of Laplace distribution called asymmetric Uniform-Laplace (AUL) distribution. In this article we present a new generalization of Esscher transformed Laplace (ETL) distribution (Sebastian and Dais, 2012) called the transmuted Esscher transformed Laplace distribution. Esscher transformed Laplace distribution is a transformed version of standard symmetric Laplace distribution and is a subclass of asymmetric Laplace distribution. This distribution belongs to one parameter exponential family.

The probability density function (pdf) and cumulative distribution function (cdf) of Esscher transformed Laplace distribution, denoted by $\operatorname{ETL}(\theta)$ are respectively given for $\theta \in(-1,1)$ as

$$
\begin{gathered}
f(x ; \theta)= \begin{cases}\frac{1-\theta^{2}}{2} e^{x(1+\theta)}, & x<0 \\
\frac{1-\theta^{2}}{2} e^{-x(1-\theta)}, & x \geq 0\end{cases} \\
\text { and } \\
F(x ; \theta)= \begin{cases}\frac{1-\theta}{2} e^{x(1+\theta)}, & x<0 \\
1-\frac{1+\theta}{2} e^{-x(1-\theta)}, & x \geq 0 .\end{cases}
\end{gathered}
$$

When $\theta \in(-1,0)$, the distribution is left skewed and when $\theta \in(0,1)$, then the distribution is right skewed.

The probability density function (pdf) and cumulative distribution function (cdf) of three parameter Esscher transformed Laplace distribution, denoted by $\operatorname{ETL}(\theta, \mu, \sigma)$ are respectively given for $\theta \in(-1,1)$ as

$$
\begin{gathered}
f(x ; \theta, \mu, \sigma)= \begin{cases}\frac{1-\theta^{2}}{2 \sigma} e^{\frac{x-\mu}{\sigma}(1+\theta)}, & x<\mu, \\
\frac{1-\theta^{2}}{2 \sigma} e^{\frac{\mu-x}{\sigma}(1-\theta)}, & x \geq \mu,\end{cases} \\
\text { and } \\
F(x ; \theta, \mu, \sigma)= \begin{cases}\frac{1-\theta}{2} e^{\frac{x-\mu}{\sigma}(1+\theta)}, & x<\mu, \\
1-\frac{1+\theta}{2} e^{\frac{\mu-x}{\sigma}(1-\theta)}, & x \geq \mu,\end{cases}
\end{gathered}
$$

were $\mu \in \mathbb{R}$ and $\sigma>0$.

In the present study we proposed the transmuted Esscher transformed Laplace (TETL) distribution as an error distribution for cDNA microarray gene expression data. Microarray is a technique widely used to asses changes in gene expression levels of thousands of genes simultaneously during several biological processes. Here level of expression of genes in one set (test) is compared with another (control) to identify differentially expressed genes. After normalization, gene expression distribution (log ratio of red and green intensity measurements) which is referred to as error distribution has heavier tails than Gaussian distribution and has asymmetry of varying degrees. The error distribution is modeled using several densities, Devika et al. (2016) used Esscher transformed 
Laplace distribution in modeling microarray data as an alternative to normal and Laplace distribution. Various authors suggested error distribution for gene expression data, asymmetric Laplace distribution (Purdom and Holmes, 2005), asymmetric type II compound Laplace (Punathumparambath et al., 2012), slash distribution with normal kernel (Punathumparambath, 2011), asymmetric slash Laplace (Punathumparambath, 2012a), skew slash $\mathrm{t}$ (Punathumparambath 2012b), Laplace mixture (Punathumparambath and Kannan, 2012), slash distribution with Cauchy kernel (Punathumparambath 2013), Double Lomax (Punathumparambath and Kulathinal, 2015) and compound exponential power (Punathumparambath, 2020).

A typical microarray data with thousands of genes show asymmetry and peakedness because a large proportion of genes are not differentially expressed, and the log-ratio of the intensities have tendency to cluster around a single point, and outliers are present. Mean, variance and skewness parameters cannot completely capture such pattern in the dataset. In the present study we introduce

the transmuted Esscher transformed Laplace (TETL) distribution which is the transmuted version of the Esscher transformed Laplace distribution. The paper is organized as follows. In Section 2, we derive the pdf, cdf, sf, hf, of, rhf, and some properties of the TETL distribution. Location scale extension of TETL and quantile function was derived in section 3. In section 4 we describe the maximum likelihood estimation of parameters using the Broyden - Fletcher - Goldfarb - Shanno (BFGS) algorithm of opt im function (Nash, 1990) in R (R-Core-Team, 2015). Simulation studies were carried out to illustrate the performance of the algorithm and is presented in section 5. In Section 6 we illustrate the applications of the proposed distribution to microarray gene expression dataset, we fitted the TETL, ETL and Gaussian distributions to microarray gene expression dataset and compared. Finally, some concluding remarks are given in section 7.

\section{Transmuted Esscher Transformed Distribution}

In this section we introduce transmuted Esscher transformed Laplace distribution. The transmutedG (T-G) family by Shaw and Buckley (2007) is defined by the cumulative distribution function (cdf) and probability density function (pdf) given by

$$
\begin{aligned}
F(x ; \lambda) & =(1+\lambda) G(x)-\lambda G(x)^{2}, \quad|\lambda| \leq 1, \\
f(x ; \lambda) & =g(x)(1+\lambda-2 \lambda G(x)), \quad-\infty<x<\infty,
\end{aligned}
$$

where $G(\cdot)$ and $g(\cdot)$ denote the $p d f$ and $c d f$, of the baseline family respectively.

Now we define probability density function of transmuted Esscher transformed Laplace (TETL) distribution.

Definition 2.1. A random variable $X$ is said to have transmuted Esscher transformed Laplace distribution with parameters $(\theta, \lambda)$, denoted by $X \sim \operatorname{TETL}(\theta, \lambda)$ if its probability density function is given by

$$
f(x ; \theta, \lambda)= \begin{cases}\frac{1-\theta^{2}}{2} e^{x(1+\theta)}\left(1+\lambda\left(1-(1-\theta) e^{x(1+\theta)}\right)\right), & x<0, \\ \frac{1-\theta^{2}}{2} e^{-x(1-\theta)}\left(1-\lambda\left(1-(1+\theta) e^{-x(1-\theta)}\right)\right), & x \geq 0,\end{cases}
$$


From the pdf given in 2.3 we can see that for $\lambda=0$ we get the Esscher transformed Laplace (ETL) distribution, $\theta=0$ we get transmuted Laplace and $\lambda=\theta=0$ we get the Laplace distribution. Probability plots of the $\operatorname{TETL}(\theta, \lambda)$ for $-1 \leq \lambda<0$ and $0 \leq \lambda \leq 1$ are presented in Figure 1 .
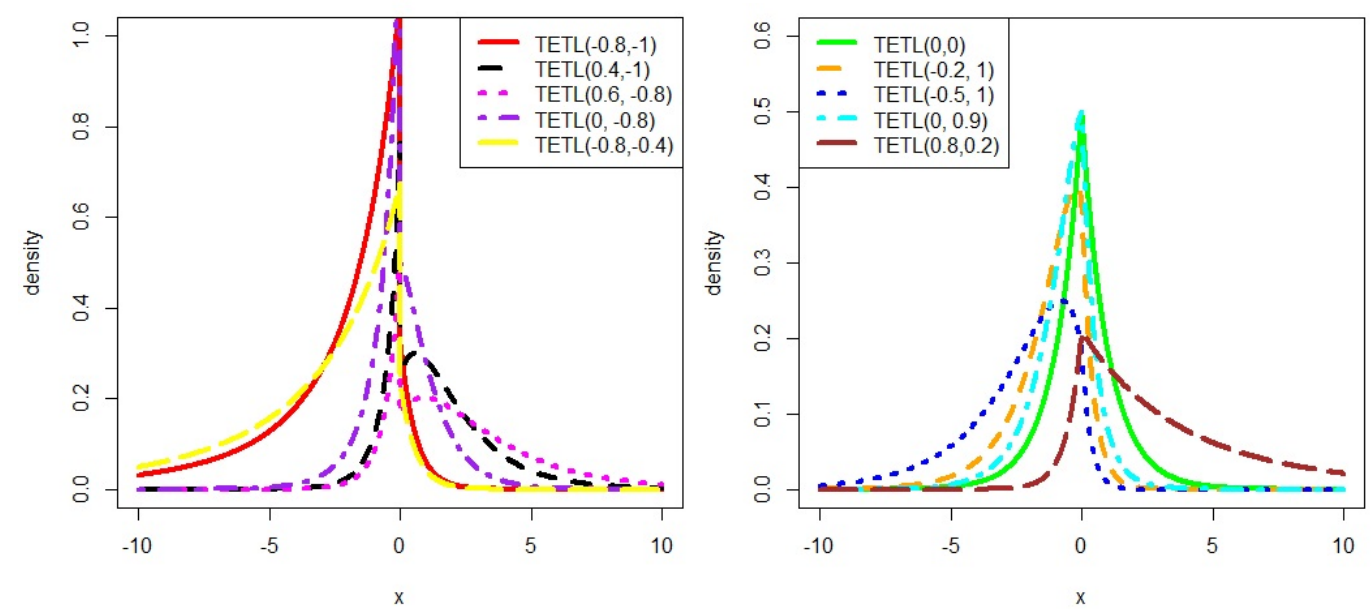

Figure 1: Densities of transmuted Esscher transformed Laplace distribution for various values of the parameters $\theta$, and $-1 \leq \lambda<0$ (left panel) and $0 \leq \lambda \leq 1$ (right panel)

From Figures 1, we can see that the distribution is positively skewed, negatively skewed, symmetric and bimodal. When $\theta=\lambda=0$, the distribution is symmetric. Also, it is very clear from the figure that TETL distribution has heavier tails than Gaussian distribution. The cumulative distribution function (cdf) of the TETL distribution is given by,

$$
F(x ; \theta, \lambda)= \begin{cases}\frac{1-\theta}{2} e^{x(1+\theta)}\left[1+\lambda\left(1-\frac{(1-\theta)}{2} e^{x(1+\theta)}\right)\right], & x<0, \\ {\left[1-\frac{1+\theta}{2} e^{-x(1-\theta)}\right]\left[1+\frac{\lambda}{2}(1+\theta) e^{-x(1-\theta)}\right],} & x \geq 0,\end{cases}
$$

where $\theta \in(-1,1)$ and $|\lambda| \leq 1$.

The survival function (sf) of the TETL distribution is given by

$$
S(x ; \theta, \lambda)= \begin{cases}1-\frac{1-\theta}{2} e^{x(1+\theta)}\left[1+\lambda\left(1-\frac{(1-\theta)}{2} e^{x(1+\theta)}\right)\right], & x<0, \\ 1-\left[1-\frac{1+\theta}{2} e^{-x(1-\theta)}\right]\left[1+\frac{\lambda}{2}(1+\theta) e^{-x(1-\theta)}\right], & x \geq 0,\end{cases}
$$

The plots of the cumulative distribution function (cdf) of the TETL distribution are given below in Figure 2 .

The hazard function (hf) of the TETL distribution is given by

$$
h(x ; \theta, \lambda)= \begin{cases}\frac{1-\theta^{2}}{2} \times \frac{e^{x(1+\theta)}\left(1+\lambda\left(1-(1-\theta) e^{x(1+\theta)}\right)\right)}{1-\frac{1-\theta}{2} e^{x(1+\theta)}\left[1+\lambda-\lambda \frac{(1-\theta)}{2} e^{x(1+\theta)}\right]}, & x<0, \\ \frac{1-\theta^{2}}{2} \times \frac{e^{-x(1-\theta)}\left(1-\lambda\left(1-(1+\theta) e^{-x(1-\theta)}\right)\right)}{1-\left[1-\frac{1+\theta}{2} e^{-x(1-\theta)}\right]\left[1+\frac{\lambda}{2}(1+\theta) e^{-x(1-\theta)}\right]}, & x \geq 0,\end{cases}
$$



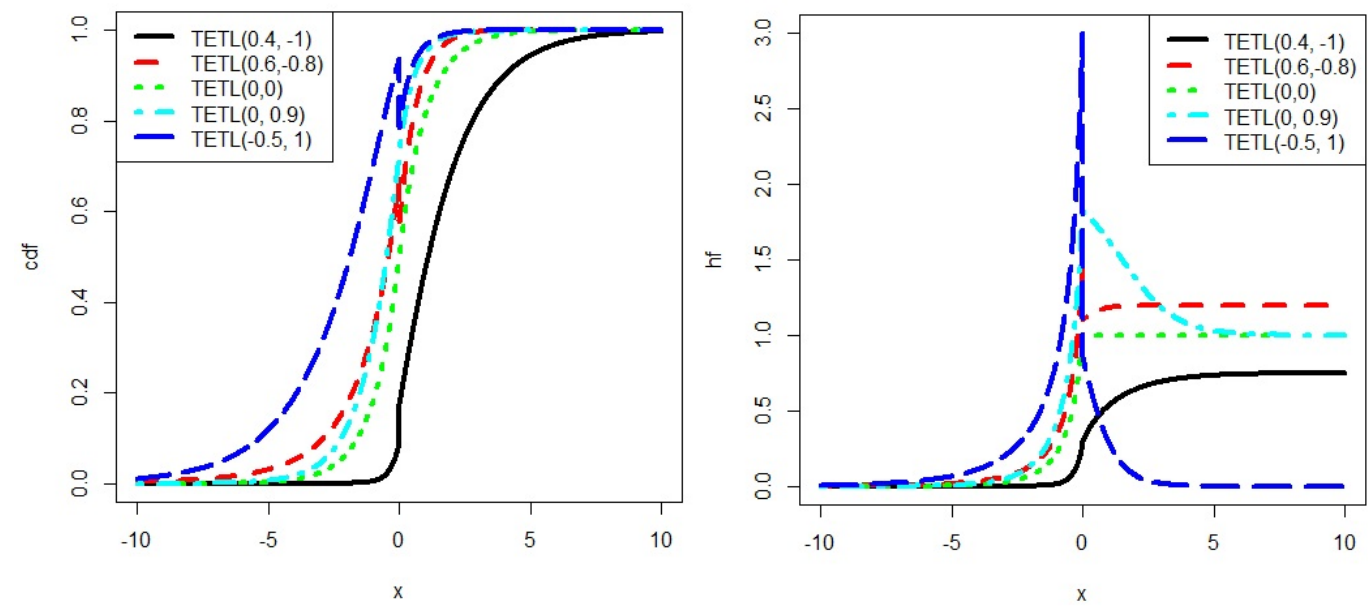

Figure 2: cdf (left panel) and hazard function (right panel) of transmuted Esscher transformed Laplace distribution for various values of the parameters $\theta$ and $\lambda$

Graph of the hazard function ( $h f)$ of $\operatorname{TETL}(\theta, \lambda)$ for various values of $\theta$ and $\lambda$ were given in Figure 2 From the plot of hazard function (Figure 2) we can observe that failure rate of the TETL is initially increasing and then decreasing over the real line. But the failure rate of Laplace distribution (green dotted line in Figure 2) is increasing in the interval $(-\infty, 0)$ and remains constant in the interval $(0, \infty)$.

The reversed hazard function ( $r h f$ ) of the TETL distribution, $r(x)=f(x) / F(x)$ is given by

$$
r(x ; \theta, \lambda)= \begin{cases}\frac{1-\theta^{2}}{2} \times \frac{e^{x(1+\theta)}\left(1+\lambda\left(1-(1-\theta) e^{x(1+\theta)}\right)\right)}{\frac{1-\theta}{2} e^{x(1+\theta)}\left[1+\lambda\left(1-\frac{(1-\theta)}{2} e^{x(1+\theta)}\right)\right]}, & x<0, \\ \frac{1-\theta^{2}}{2} \times \frac{e^{-x(1-\theta)}\left(1-\lambda\left(1-(1+\theta) e^{-x(1-\theta)}\right)\right)}{\left[1-\frac{1+\theta}{2} e^{-x(1-\theta)}\right]\left[1+\frac{\lambda}{2}(1+\theta) e^{-x(1-\theta)}\right]}, & x \geq 0 .\end{cases}
$$

Graph of the reversed hazard function ( $r h f)$ of $\operatorname{TETL}(\theta, \lambda)$ for various values of $\theta$ and $\lambda$ were given in Figure 3. From the Figure 3 we can observe that the reverse failure rate of the TETL exhibits both increasing and decreasing behavior over the real line. The reverse failure rate of Laplace distribution (green dotted line in Figure 3 ) is decreasing in the positive support and remains constant in the negative support of the random variable.

The odds function (of) of the TETL distribution $O(x)=\frac{F(x)}{1-F(x)}$, is given by

$$
O(x ; \theta, \lambda)= \begin{cases}\frac{\frac{1-\theta}{2} e^{x(1+\theta)}\left[1+\lambda\left(1-\frac{(1-\theta)}{2} e^{x(1+\theta)}\right)\right]}{1-\frac{1-\theta}{2} e^{x(1+\theta)}\left[1+\lambda\left(1-\frac{(1-\theta)}{2} e^{x(1+\theta)}\right)\right]}, & x<0, \\ \frac{\left[1-\frac{1+\theta}{2} e^{-x(1-\theta)}\right]\left[1+\frac{\lambda}{2}(1+\theta) e^{-x(1-\theta)}\right]}{1-\left[1-\frac{1+\theta}{2} e^{-x(1-\theta)}\right]\left[1+\frac{\lambda}{2}(1+\theta) e^{-x(1-\theta)}\right]}, & x \geq 0 .\end{cases}
$$

Graph of the odds function (of) of the $\operatorname{TETL}(\theta, \lambda)$ for various values of $\theta$ and $\lambda$ are given in Figure 3. 

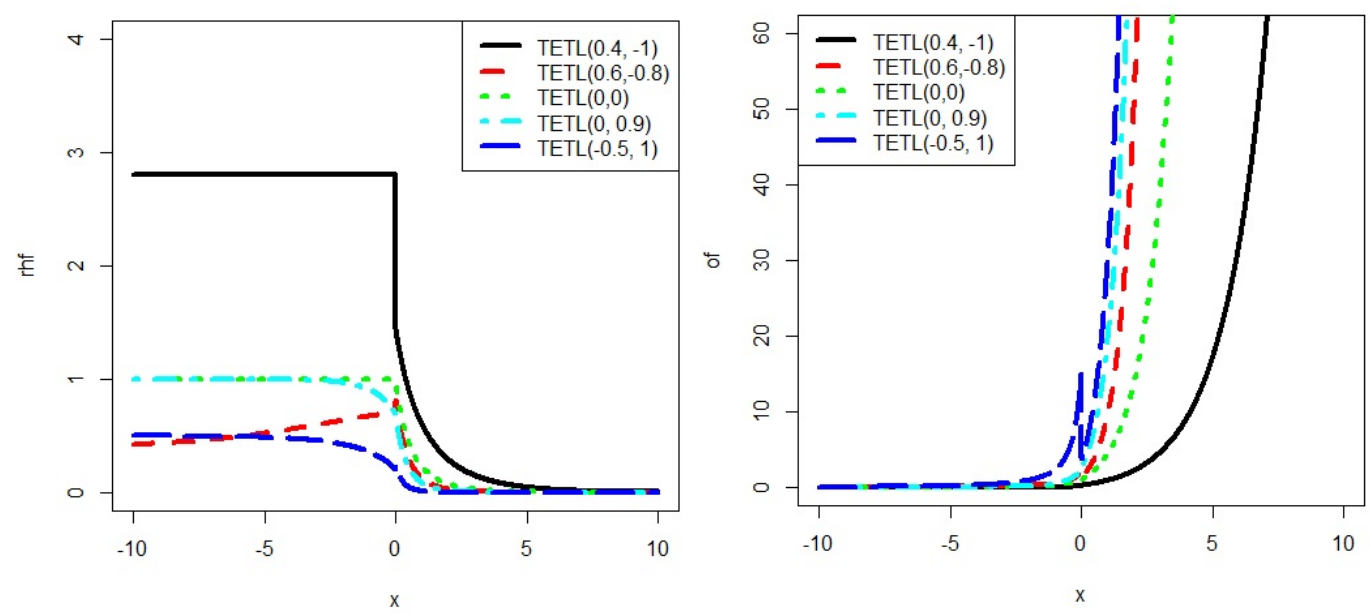

Figure 3: Reverse hazard function (left panel) and odds function (right panel) of transmuted Esscher transformed Laplace distribution for various values of parameters $\theta$ and $\lambda$

\subsection{Moments}

For $r>0$, the $r^{t h}$ raw moment of TETL is given by

$$
\begin{aligned}
\mu_{r}^{\prime} & =E\left(X^{r}\right) \\
& =\frac{\left(1-\theta^{2}\right) \Gamma(r+1)}{2}\left[\frac{(-1)^{r}\left(1+\lambda-\lambda(1-\theta) / 2^{(r+1)}\right)}{(1+\theta)^{r+1}}+\frac{1-\lambda+\lambda(1+\theta) / 2^{(r+1)}}{(1-\theta)^{r+1}}\right],
\end{aligned}
$$

$\theta \in(-1,1)$. For $r=1$ we get the mean. The mean of the $\operatorname{TETL}(\theta, \lambda)$ is given by

$$
\text { Mean }=E(X)=\frac{8 \theta-3 \lambda-\lambda \theta^{2}}{4\left(1-\theta^{2}\right)}
$$

The moment generating function (m.g.f) of the TETL for $\theta \in(-1,1)$ and $t \in(-4,4)$ is given by

$$
M_{X}(t)=\frac{1-\theta^{2}}{2}\left[\frac{1+\lambda}{(t+1+\theta)}-\frac{\lambda(1-\theta)}{(t+2+2 \theta)}+\frac{1-\lambda}{(1-\theta-t)}+\frac{\lambda(1+\theta)}{(2-2 \theta-t)}\right] \text {. }
$$

\section{Four Parameter Transmuted Esscher transformed Laplace dis- tribution}

In this section we define four parameter transmuted Esscher transformed Laplace distribution. The pdf of the four parameter TETL distribution with parameters $(\theta, \mu, \sigma, \lambda)$ are respectively given below. 


$$
f(x ; \theta, \mu, \sigma, \lambda)= \begin{cases}\frac{1-\theta^{2}}{2 \sigma} e^{\frac{x-\mu}{\sigma}(1+\theta)}\left(1+\lambda\left(1-(1-\theta) e^{\frac{x-\mu}{\sigma}(1+\theta)}\right)\right), & x<\mu, \\ \frac{1-\theta^{2}}{2 \sigma} e^{\frac{\mu-x}{\sigma}(1-\theta)}\left(1-\lambda\left(1-(1+\theta) e^{\frac{\mu-x}{\sigma}(1-\theta)}\right)\right), & x \geq \mu,\end{cases}
$$

and the cdf of the four parameter TETL distribution with parameters $(\theta, \mu, \sigma, \lambda)$ are respectively given below in Equation 3.2 .

$$
F(x ; \theta, \mu, \sigma, \lambda)= \begin{cases}\frac{1-\theta}{2} e^{\frac{x-\mu}{\sigma}(1+\theta)}\left(1+\lambda-\lambda \frac{(1-\theta)}{2} e^{\frac{x-\mu}{\sigma}(1+\theta)}\right), & x<\mu, \\ {\left[1-\frac{1+\theta}{2} e^{\frac{\mu-x}{\sigma}(1-\theta)}\right]\left(1+\frac{\lambda}{2}(1+\theta) e^{\frac{\mu-x}{\sigma}(1-\theta)}\right),} & x \geq \mu,\end{cases}
$$

where $\theta \in(-1,1), \mu \in \mathbb{R}, \sigma>0$ and $|\lambda| \leq 1$.

Graphs of pdf of $\operatorname{TETL}(\theta, \mu, \sigma, \lambda)$ for $\theta=-0.5,0.5, \mu=-2,0,2, \sigma=0.51 .5$ and $\lambda=-1,1$ is given in Figure 4.

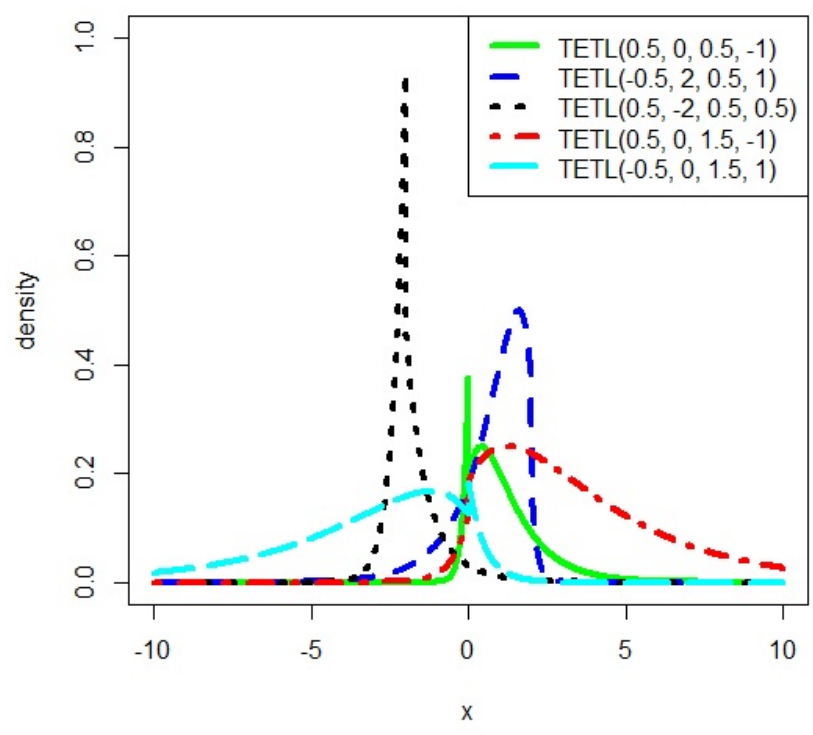

Figure 4: Densities of transmuted Esscher transformed Laplace distribution for various values of parameters $\theta, \mu, \sigma, \lambda$.

\subsection{Quantile Function}

The $q^{\text {th }}$ quantile function of the TETL distribution is given by

$$
\xi_{q}=\left\{\begin{array}{l}
\mu+\frac{\sigma}{1+\theta} \ln \left(\frac{\left[1+\lambda-\sqrt{(1+\lambda)^{2}-4 \lambda q}\right]}{\lambda(1-\theta)}\right), q \in\left(0, \frac{1-\theta}{4}[2+\lambda(1+\theta)]\right], \\
\mu-\frac{\sigma}{1-\theta} \ln \left(\frac{\left[\lambda-1+\sqrt{(1+\lambda)^{2}-4 \lambda q}\right]}{\lambda(1+\theta)}\right), q \in\left(\frac{1-\theta}{4}[2+\lambda(1+\theta)], 1\right] .
\end{array}\right.
$$


The median of TETL is obtained by putting $q=1 / 2$ and is given by

$$
\text { Median }=\xi_{\frac{1}{2}}=\left\{\begin{array}{l}
\mu+\frac{\sigma}{1+\theta} \ln \left(\frac{\left[1+\lambda-\sqrt{1+\lambda^{2}}\right]}{\lambda(1-\theta)}\right), \theta, \lambda \in(-1,0), \\
\mu-\frac{\sigma}{1-\theta} \ln \left(\frac{\left[\lambda-1+\sqrt{1+\lambda^{2}}\right]}{\lambda(1+\theta)}\right), \theta, \lambda \in(0,1) .
\end{array}\right.
$$

The $c d f$ and $q f$ can be useful for goodness-of-fit and simulation purposes. For $q=\frac{1-\theta}{4}[2+$ $\lambda(1+\theta)]$, the $q^{\text {th }}$ quantile is given by $\xi_{q}=\mu$. Hence, for given $\kappa$ the location parameter is given by $\hat{\mu}=\xi_{\left[\frac{1-\theta}{4}[2+\lambda(1+\theta)]\right.}$.

The skewness and kurtosis can be defined based on the quantile function. The Galton's skewness (Galton, 1883) and the Moors kurtosis (Moors, 1988) coefficients are, respectively

$$
S=\frac{\xi_{[6 / 8]}-2 \xi_{[4 / 8]}+\xi_{[2 / 8]}}{\xi_{[6 / 8]}-\xi_{[2 / 8]}} \text { and } K=\frac{\xi_{[7 / 8]}-\xi_{[5 / 8]}+\xi_{[3 / 8]}-\xi_{[1 / 8]}}{\xi_{[6 / 8]}-\xi_{[2 / 8]}} .
$$

The distribution is symmetric, right (or left) skewed for $S=0, S>0$ (or $S<0$ ), respectively. As the value of kurtosis increases, the tail heaviness of the distribution increases.

\section{Estimation}

In this section we study the problem of estimating four unknown parameters, $\Theta=(\theta, \mu, \sigma, \lambda)^{\prime}$, of the TETL distribution. To estimate the parameter $\mu$ we use the quantile estimation. The method of maximum likelihood estimation can be employed to estimate $\Theta$. To estimate $\Theta$ using maximum likelihood estimation where the likelihood function is maximized to estimate the unknown parameters. We describe this method briefly as follows. Let $X=\left(X_{1}, \ldots, X_{n}\right)^{\prime}$ be independent and identically distributed samples from an TETL distribution with parameters $\Theta$ and $X_{(1)}, \ldots, X_{(n)}$ be the ordered sample observations. Assume $X_{(r)}<\mu<X_{(r+1)}$, for $r=1,2, \ldots, n$.

The log-likelihood function of the data $X$ takes the form

$$
\begin{aligned}
L(\Theta)= & -n \log 2-n \log \sigma+n \log \left(1-\theta^{2}\right)+\sum_{I_{1}} \frac{x_{i}-\mu}{\sigma}(1+\theta) \\
& -\sum_{I_{2}} \frac{x_{i}-\mu}{\sigma}(1-\theta)+\sum_{I_{1}} \log \left(G_{i}\right)+\sum_{I_{2}} \log \left(H_{i}\right),
\end{aligned}
$$

were

$$
\begin{aligned}
& G_{i}=1+\lambda\left(1-(1-\theta) e^{\frac{x_{i}-\mu}{\sigma}(1+\theta)}\right), \\
& H_{i}=1-\lambda\left(1-(1+\theta) e^{\frac{\mu-x_{i}}{\sigma}(1-\theta)}\right),
\end{aligned}
$$

$\sum_{I_{i}}$ denotes the summation over the set $I_{i}$ such that

$$
\begin{aligned}
& I_{1}=\left\{j: X_{(j)}<\mu, \text { for } j=1,2, \ldots, n\right\} \text { and } \\
& I_{2}=\left\{j: X_{(j)}>\mu, \text { for } j=1,2, \ldots, n\right\},
\end{aligned}
$$


respectively.

MLEs of $\theta, \sigma$ and $\lambda$ for given $\mu=\hat{\mu}$ are obtained by solving the score equations iteratively. In this paper MLEs of $(\theta, \mu, \sigma, \lambda)$ were obtained by maximizing the log likelihood using the optim function of the R statistical software, applying the Broyden - Fletcher - Goldfarb - Shanno (BFGS) algorithm (Nash, 1990) in R (R-Core-Team, 2015). Estimates of the standard errors were obtained by inverting the numerically differentiated information matrix at the maximum likelihood point.

\section{Simulation Study}

In this section we perform the simulation studies for various choices of parameters to evaluate the performance of the estimation procedure. We generated 1000 samples, each of size $n=50,100,250$ from the TETL distribution for $\theta=-0.5,0.5, \mu=0.05, \sigma=(0.5,1)$ and $\lambda=(-0.5,0,0.5)$ and then applied the algorithm to obtain the MLEs of the parameters. To generate $\operatorname{TET} L(\theta, \mu, \sigma, \lambda)$ the following algorithm was used:

- Input number of replications $N=1000$.

- Give various values for sample size $\mathrm{n}$ and for parameters .

- Generate random samples from uniform $U \sim U(0,1)$.

- Generate random samples from TETL using quantile function given in Equation 3.3

- Compute MLEs of the parameters $\theta, \mu, \sigma$, and $\lambda$ by applying the BFGS algorithm in R.

- Repeat the steps 3 to 5, $\mathrm{N}$ times.

- Compute the estimate of the MLE's, Standard Error (SE) and sample standard deviations over the replications, of the parameters.

The results from 1000 replications are presented in Table 1. It is clear from Table 1 that the estimation algorithm works satisfactorily for various choices of parameters and the asymptotic standard errors of the maximum likelihood estimators agree well with the sample standard deviations over the replications.

We also checked our algorithm for various choices of initial values of parameters and sample size $n$. For arbitrary initial values, the number of iterations needed for the iterative methods to converge were larger compared to that required when the parameters were initialized by their moment estimates. We also simulated data for varying parameters, especially towards boundary regions. When $\sigma$ is very small, comparatively larger number of iterations were needed to achieve reasonable convergence. 


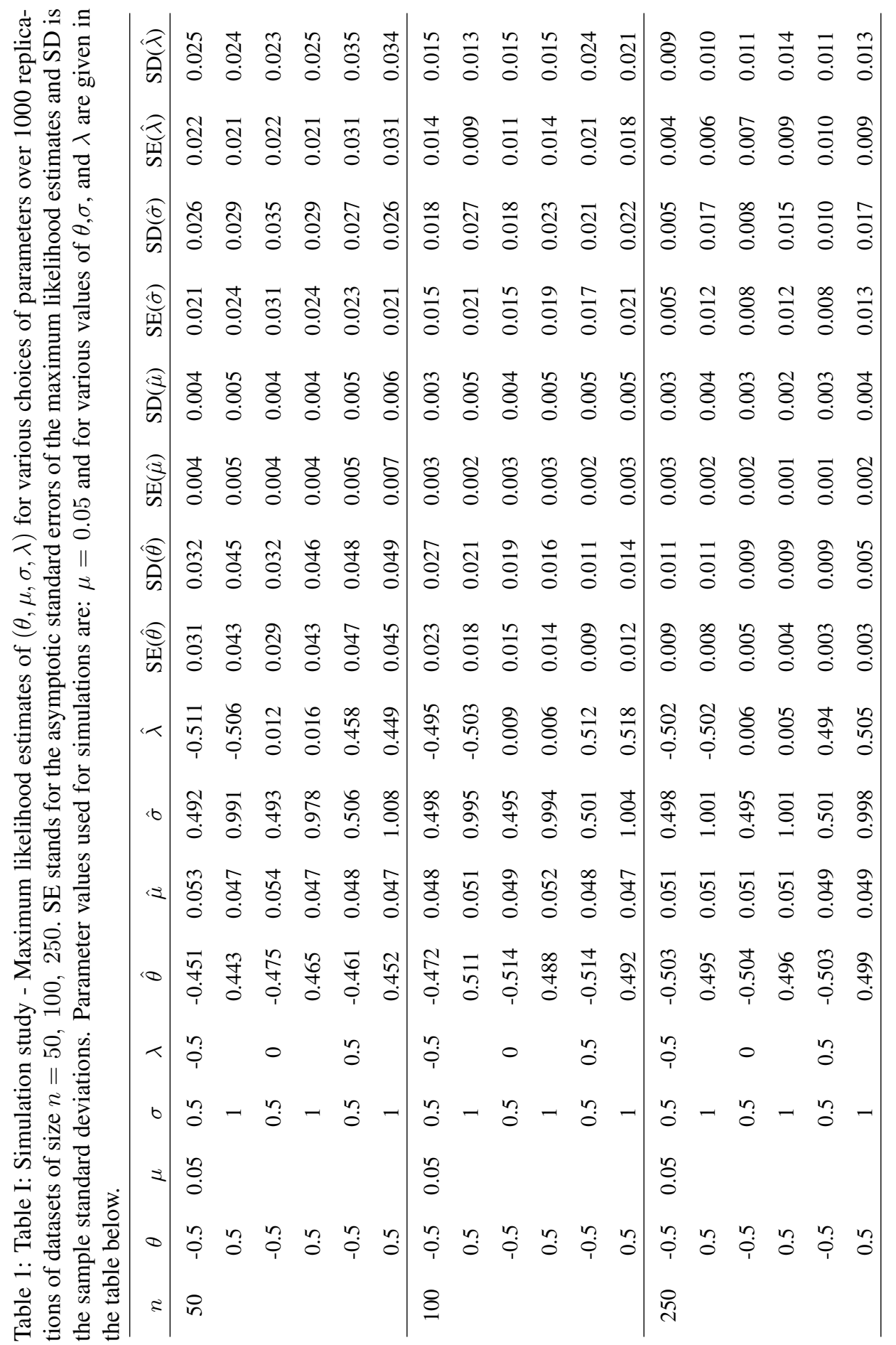




\section{Application}

In this section we illustrate the application of TETL to microarray gene expression dataset. We downloaded the cDNA dual dye microarray data sets with Experiment id-51401 from the Stanford Microarray Database. Each array chip contains approximately 42000 human cDNA elements, representing over 30000 unique genes. The dataset was normalized using (Lowess) locally weighted linear regression method (Cleveland and Delvin, 1988). This method is capable of removing intensity dependence in $\log _{2}\left(R_{i} / G_{i}\right)$ values and it has been successfully applied to microarray data (Yang et al. 2002), where $R_{i}$ is the red dye intensity and $G_{i}$ is the green dye intensity for the $i^{t h}$ gene. Figure 5 present the box plots of intensities before and after Lowess normalization. The descriptive statistics for microarray dataset with 41472 observations is given below in Table 2 and from the Table 2 we can see that the microarray dataset with Experiment id-51401 is positively skewed and highly peaked.

Here we fitted Gaussian, ETL and TETL distributions to log-transformed normalized intensities $\log _{2}\left(R_{i} / G_{i}\right)$ from the microarray dataset mentioned above. We obtained maximum likelihood estimates and their asymptotic standard errors for the parameters of $\operatorname{TETL}(\theta, \mu, \sigma, \lambda), \operatorname{ETL}(\theta, \mu, \sigma)$ and Gaussian $N\left(\mu, \sigma^{2}\right)$ distributions (see Table 3 and Figure 6 .

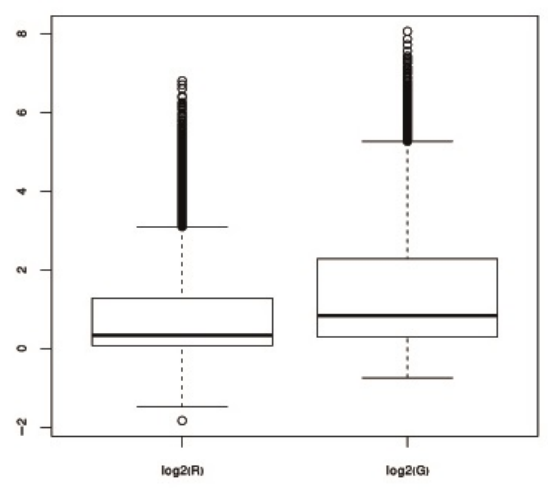

(a)

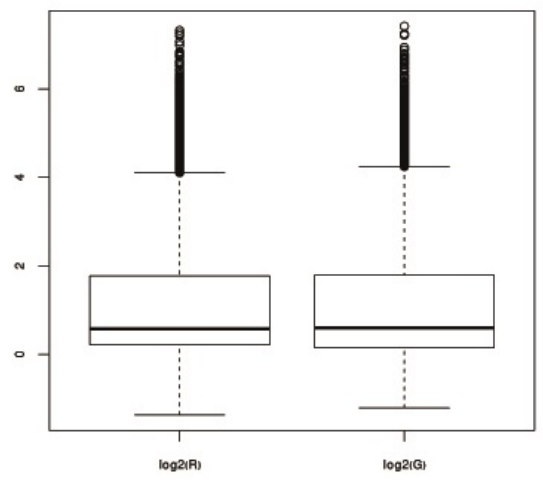

(b)

Figure 5: Box plots of intensities from microarray Experiment Id 51401 (a) Before normalization, (b) After loess normalization. 
Table 2: Descriptive statistics for microarray dataset)

\begin{tabular}{lcccccc}
\hline Min & Max & Mean & Median & SD & Skewness & Kurtosis \\
\hline-5.809 & 6.773 & 0.006 & 0.032 & 0.798 & 0.490 & 10.639 \\
\hline
\end{tabular}

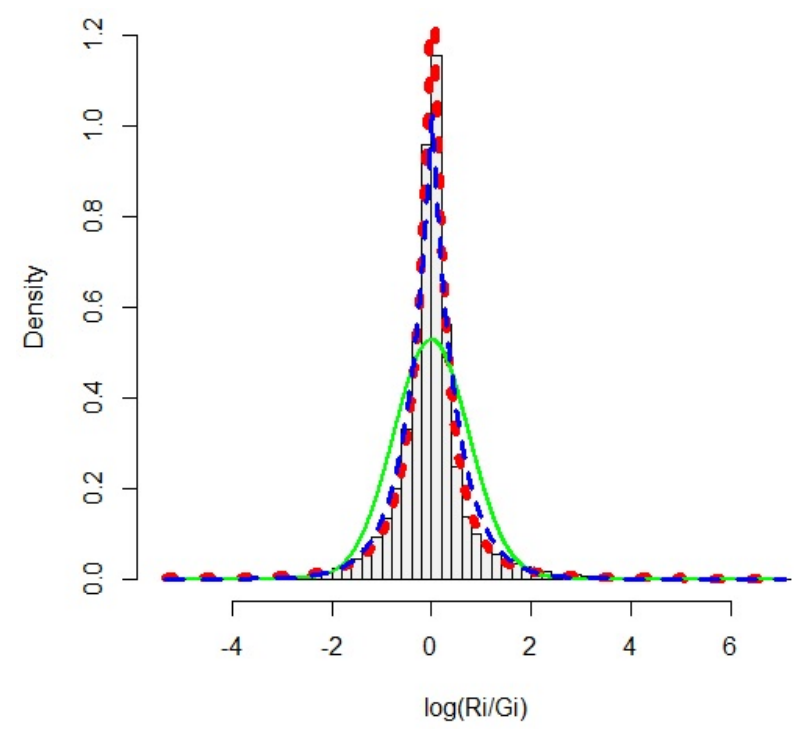

Figure 6: Fitted transmuted Esscher transformed Laplace (TETL)(red dotted line), Esscher transformed Laplace (ETL)(blue dashed) and Gaussian (green line) distributions (evaluated at MLEs) for the microarray data from Experiment id-51401.

We used Akaike's Information Criterion (AIC) (Akaike (1973); Burnham and Anderson (1998)) and Bayesian Information Criterion (BIC) (Schwarz, 1978) to assess the appropriateness of TETL over the ETL and Gaussian distributions. The $A I C$ and $B I C$ are given by

$$
A I C=-2 \log L+2 K \text { and } B I C=-2 \log L+K \log (n),
$$

where $\log L=\log L_{f}\left(\hat{\theta} \mid x_{1}, \ldots, x_{n}\right)$ is the $\log$-likelihood of the data $x_{1}, \ldots, x_{n}$ under the probability distribution $f, K$ is the number of parameters being estimated, $\hat{\theta}$ is the maximum likelihood estimate of the parameters of $f$ and $n$ is the sample size. In most cases $A I C$ and $B I C$ are of similar nature and give consistent results for model selection.

A smaller value of $A I C$ or $B I C$ indicates a better fit. Table 3 shows the $A I C$ and $B I C$ for the $\operatorname{TETL}(\theta, \mu, \sigma, \lambda), \operatorname{ETL}(\theta, \mu, \sigma)$ and $N\left(\mu, \sigma^{2}\right)$ distributions for the microarray dataset examined. The TETL $(\theta, \mu, \sigma, \lambda)$ distribution had a lower $A I C$ and $B I C$ for the microarray gene expression dataset. A smaller value of $A I C$ and $B I C$ indicates a better fit, and hence, $T E T L$ fit the data better than $E T L$ or Gaussian distributions. 
Table 3: Microarray data analysis - maximum likelihood estimates and their asymptotical standard deviations, Akaike's Information Criterion (AIC) and Bayesian Information Criterion (BIC) for TETL, ETL and Gaussian distributions.

\begin{tabular}{lcccccc}
\hline & $\hat{\theta}$ & $\hat{\mu}$ & $\hat{\sigma}$ & $\hat{\lambda}$ & $A I C$ & $B I C$ \\
\hline TETL & $0.064(0.018)$ & $0.009(0.003)$ & $0.399(0.003)$ & $0.261(0.021)$ & 87511 & 87546 \\
ETL & $0.055(0.025)$ & $0.011(0.003)$ & $0.405(0.004)$ & - & 88509 & 88533 \\
Gaussian & - & $0.006(0.001)$ & $0.797(0.004)$ & - & 98990 & 99007 \\
\hline
\end{tabular}

\section{Conclusion}

Sebastian and Dais (2012) introduced Esscher transformed Laplace distribution and modeled exchange rate data using Esscher transformed Laplace distribution. Bindu and Dais (2017) introduced first Order Moving Average Model with Esscher Transformed Laplace Innovations. A generalization of Esscher transformed Laplace distribution through the quadratic rank transmutation map introduced in this paper is useful in analyzing datasets that are asymmetric, leptokurtic, unimodal, bimodal and deviate considerably from the classical symmetric distributions such as normal, Laplace, etc.

Devika et al. (2016) used Esscher transformed Laplace distribution in modeling microarray data as an alternative to normal and Laplace distribution. In this work, we have proposed a new statistical model for the distribution of differential gene expression, which is a heavy tailed generalization of Laplace distribution. We found that TETL fit the microarray data better than ETL or Gaussian distributions. TETL is asymmetric, peaked and heavy-tailed; hence it is a proper distribution to accommodate outliers in the data. The probability distribution presented in this paper will be very useful in estimation and detection problems involving gene expression data. This distribution may be useful for financial modelling, since this distribution capture skewness, heavier tails and kurtosis present in the financial datasets.

\section{Acknowledgements}

The author is grateful to the Department of Science and Technology, Science and Engineering Research Board (SERB), Government of India, New Delhi, for financial assistance under Core Research Grand, Project No. CRG/2018/004468.

\section{References}

Akaike, H. (1973), Information theory and an extension of the maximum likelihood principle In Breakthroughs in Statistics (Kotz and Johnson eds.), vol. I, Springer Verlag, New York.

Arezoomand, F., Yarmohammadi, M., and Mahmoudvand, R. (2018), "Asymmetric Uniform- 
Laplace Distribution: Properties and Applications," Journal of The Iranian Statistical Society, $17,119-140$.

Aryal, G. and Tsokos, C. (2009), "On the transmuted extreme value distribution with application," Nonlinear Analysis: Theory, Methods and Applications, 71, 1401-1407.

- (2011), "Transmuted Weibull distribution: A generalization of the Weibull probability distribution," European Journal of Pure and Applied Mathematics, 4, 89-102.

Bindu, K. and Dais, G. (2017), "A first order moving average model with esscher transformed Laplace innovations," International Journal of Statistics and Systems, 12(4), 797-802.

Bourguignon, M., Leáo, J., Leiva, V., and Santos-Neto, M. (2017), "The transmuted BirnbaumSaunders distribution," REVSTAT Statistical Journal, 15(4), 601-628.

Burnham, K. and Anderson, D. (1998), Model selection and Inference, Springer.

Cleveland, W. S. and Delvin, S. J. (1988), "Locally weighted regression: an approach to regression analysis by local fitting," Journal of American Statistical Association, 83(403), 596-610.

Devika, S., Sebastian, G., and Jeyaseelan, L. (2016), "Applications of Esscher Transformed Laplace Distribution in Microarray Gene Expression Data," Journal of Modern Applied Statistical Methods, 15(1), 616-634.

Galton, F. (1883), Inquiries into human faculty and its development, London Macmillan and Co.

Hady, D. and Shalabi, R. (2016), "Transmuted Laplace Distribution: Properties and Applications," American Journal of Applied Mathematics and Statistics, 4(3), 94-98.

Merovci, F. (2013), “Transmuted Lindley distribution," International Journal of Open Problems in Computer Science Mathematics, 6.

Merovci, F. and Pukab, L. (2014), “Transmuted Pareto Distribution,” ProbStat, 7(1), 1-11.

Moors, J. J. (1988), “A quantile alternative for kurtosis," Journal of the Royal Statistical Society, Series D (The Statistician), 37(1), 25-32.

Nash, J. C. (1990), Compact Numerical Methods for Computers. Linear Algebra and Function Minimisation, Adam Hilger.

Punathumparambath, B. (2011), "A new family of skewed slash distribution generated by normal kernel," Statistica, 71, 345-353.

- (2012a), "The multivariate asymmetric slash Laplace distribution and its applications," Statistica, $72,235-249$.

- (2012b), "The multivariate skew-slash t and skew-slash Cauchy distributions," Model Assisted Statistics and Applications, 7, 33-40. 
- (2013), "A new family of skewed slash distribution generated by Cauchy kernel," Communications in Statistics-Theory and Methods, 42, 2351-2361.

- (2020), "Compound Exponential Power Distribution and its Application to Microarray data," International Journal of Scientific Engineering and Research, 8, 4-8.

Punathumparambath, B. and Kannan, V. M. (2012), "Two component mixed Laplace model for microarray gene expression data," JP Journal of Fundamental and Applied Statistics, 2, 15-25.

Punathumparambath, B. and Kulathinal, S. (2015), "Double Lomax distribution and its applications," Statistica, 75, 331-342.

Punathumparambath, B., Kulathinal, S., and Sebastian, G. (2012), "Asymmetric type II compound Laplace distribution and its application to microarray gene expression," Computational Statistics and Data analysis, 56, 1396-1404.

Purdom, E. and Holmes, S. (2005), "Error distribution for gene expression data," Statistical Applications in Genetics and Molecular Biology, 4, Article 16.

R-Core-Team (2015), R: A language and environment for statistical computing, R Foundation for Statistical Computing, Vienna, Austria.

Schwarz, G. (1978), "Estimating the dimension of a model," Annals of Statistics, 6, 461-464.

Sebastian, G. and Dais, G. (2012), "Esscher transformed Laplace distribution and its applications," Journal of Probability and Statistical Science, 10, 135-152.

Shaw, W. T. and Buckley, I. R. C. (2007), The Alchemy of Probability Distributions: Beyond GramCharlier and Cornish-Fisher Expansions and Skew-Normal or Kurtotic-Normal Distributions, Research report.

Yang, Y. H., Dudoit, S., Luu, P., Lin, D. M., Peng, V., Ngai, J., and Speed, T. (2002), "Normalization for cDNA microarray data: a robust composite method addressing single and multiple slide systematic variation," Nucleic Acids Reseach, 30, e 15.

Received: August 20, 2020

Accepted: January 26, 2021 